\title{
Microbial Intervention as a Novel Target in Treatment of Non-Alcoholic Fatty Liver Disease Progression
}

\author{
Youlian Zhou ${ }^{a, b}$ Tiying Zheng ${ }^{b} \quad$ Huiting Chen ${ }^{a, b} \quad$ Yongqiang Lia,b Hongli Huang ${ }^{a, b}$ \\ Wenji Chen ${ }^{a, b}$ Yanlei Du ${ }^{a, b}$ Jie He $e^{a, b}$ Yuyuan Li ${ }^{a, b}$ Jie Cao ${ }^{a, b}$ Yuqiang Nie \\ Yongjian Zhou a,b
}

aDepartment of Gastroenterology, Guangzhou Digestive Disease Center, Guangzhou First People's Hospital, the Second Affiliated Hospital of South China University of Technology, Guangzhou, ${ }^{b}$ Department of Gastroenterology, Guangzhou Digestive Disease Center, Guangzhou First People's Hospital, Guangzhou Medical University, Guangzhou, China

\section{Key Words}

Nonalcoholic fatty liver disease $\cdot \operatorname{NAFLD} \cdot$ Gut microbiota $•$ Adiponectin $•$ And methylation

\begin{abstract}
Background/Aims: Emerging evidence suggests a close link between gut microbiota and non-alcoholic fatty liver disease (NAFLD). In this study, we aimed to investigate the association between gut microbiota and the DNA methylation of adiponectin (an adipocytespecific adipocytokine) in rats, following diet-induced NAFLD. Methods: 50 male SD rats were randomly divided into five groups with or without a high fat diet (HFD), antibiotics, and probiotics, in order to establish an imbalanced gut microbiota and probiotic treatment model in NAFLD rats. After 13 weeks of treatment, blood, liver, and cecal tissue samples were collected. Serum lipids, liver function indexes by biochemical analyzers, and changes in liver pathology with hematoxylin-eosin $(\mathrm{HE})$ and masson staining were detected. Furthermore, the serum adiponectin by enzyme-linked immunosorbent assay (ELISA) and liver adiponectin methylation levels in the promoter regions by pyrophosphate sequencing were determined. High throughput Illumina sequencing targeted microbial $16 \mathrm{~S}$ genes, bioinformatics and statistical analysis identified cecal-associated gut microbiota. Results: HFD with antibiotic exposure showed the most severe steatohepatitis and a severe gut microbiota alteration. Reduced bacterial diversity was also seen and the abundances of Firmicutes, Lactobacillus, Cyanobacteria, Acidobacteria, Chlamydiae, Chlamydiales, Rubrobacteria, Verrucomicrobia, Blautia, Shewanella, Bacteroides, Bacteroides acidifaciens, and Bacteroides uniformis, were shown to be partly reversed by probiotic treatment. Decreased serum adiponectin levels and increased DNA methylation levels of adiponectin promoter regions were also markedly associated with the NAFLD progression during gut microbiota alteration. Conclusion: Our

Y. Zhou, T. Zheng, H. Chen and Y. Li contributed equally to this article.

\begin{tabular}{ll}
\hline Yongjian Zhou & Dept. of Gastroenterology, Guangzhou Digestive Disease Center, Guangzhou First People's Hospital, \\
& the Second Affiliated Hospital of South China Univ. of Tech, Guangzhou Key Lab. of Digestive Disease, \\
& Guangzhou Med. Univ., No. 1 Panfu Road, Guangzhou 510180, (China); E-Mail yjzhou@gzhmu.edu.cn
\end{tabular}
\end{abstract}




\section{Cellular Physiology Cell Physiol Biochem 2018;51:2123-2135

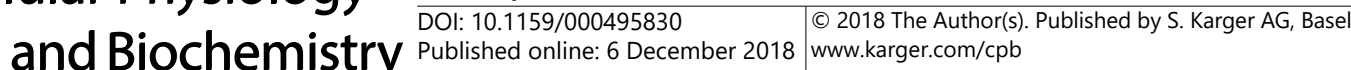 \\ Zhou et al.: Microbiota in Non-Alcoholic Fatty Liver Disease}

results suggested that both gut microbiota alteration and adiponectin variability may be drivers of NAFLD progression and that targeting the gut microbiota, such as via administration of a probiotic, may delay NAFLD progression via adiponectin.

(C) 2018 The Author(s)

Published by S. Karger AG, Basel

\section{Introduction}

Nonalcoholic fatty liver disease (NAFLD) is a metabolic syndrome characterized by hepatic fat accumulation in the absence of significant alcohol consumption [1]. As the prevalence of obesity increases, the numbers of NAFLD patients over the past 20 years have also dramatically increased. The increases of NAFLD prevalence and obesity have been identified as a major contributor to chronic liver disease (CLD) worldwide [2-4]. NAFLD includes diseases ranging from the less severe simple steatosis (NAFL) to severe inflammatory steatohepatitis (NASH). NAFL is generally regarded as a benign state, whereas NASH is classified as severe NAFLD and has a prevalence of $2-5 \%$ among NAFLD populations [5]. NASH is an important risk factor for the development of liver cirrhosis, liver carcinoma, and other complications of portal hypertension [6-9], the pathology is characterized by hepatocyte injury, inflammation, and various degrees of fibrosis in addition to steatosis [10]. Recently reported data have improved the understanding of NAFLD pathogenesis, yet key factors contributing to the progression of NAFLD from NAFL towards NASH remain only partially elucidated.

Adiponectin, a protein hormone secreted by adipose tissues, is closely associated with the pathogenesis and development of NAFLD. Adiponectin plays an important biological role, in combination with the adiponectin receptors of the liver, in increasing insulin sensitivity, resistance to liver fat deposition, inhibition of inflammatory response, stabilization of oxidative stress, and fibrosis. We have confirmed in previous studies that insulin resistance (IR) in patients with NAFLD is associated with the low expression of adiponectin among adipose tissues. Further, the adiponectin associated single nucleotide polymorphism (SNP) sites are also closely related to susceptibility of NAFLD disease progression [11,12]. Further studies in epigenetics suggest the roles of DNA methylation in NAFLD pathogenesis merit continued investigation. DNA methylation refers to the covalent binding of the fifth carbon atom of cytosine in S-adenosine methyl to a DNA molecule under the action of DNA methyl transferase (DNMTs), which is then modified to 5-methyl cytosine [13]. In the physiological state methylation of DNA promoter regions play an important role in cell differentiation, growth, and development. When methylation occurs abnormally it often hinders the binding of a transcription factor with a promoter, leading to decreased and potentially silenced gene transcription. In a recent study of the association between maternal glucose concentration and the DNA methylation level in the placenta adiponectin gene, the frequency of adiponectin DNA methylation was correlated with maternal blood glucose levels during pregnancy, insulin resistance, and maternal circulating adiponectin concentrations [14]. Many genes have been found to be highly methylated in the presence of hepatocellular carcinoma [15], but little known about methylation as it relates to NAFLD. Therefore, it is of great value to examine the potential for DNA methylation of adiponectin to slow the progression of NASH.

The human gut microbiota contains more than 100 fold genes than its host [16] and has been suggested to be an important environmental factor involved in the pathogenesis of NAFLD $[17,18]$. There is an anatomical link between the intestine and liver via the hepatic portal system. Based on the connection between intestine and liver, also termed the gutliver axis, gut microbiota and their metabolic products may influence liver pathology [19]. $\mathrm{Li}$, et al., was first to report that the manipulation of gut microbiota in animal models could influence obesity-related fatty liver disease [20]. Moreover in NAFLD, an imbalanced gut microbiota can affect lipid metabolism in various ways resulting in an attack on the liver from two fronts. Vähämiko, et al., suggested that administering a probiotic supplement during pregnancy may affect the DNA methylation status of certain obesity and weight gainrelated gene in both mothers and their children [21]. 


\section{Cellular Physiology Cell Physiol Biochem 2018;51:2123-2135 \begin{tabular}{l|l|l} 
and Biochemistry Published online: 6 December 2018 & $\begin{array}{l}\text { ○ } 2018 \text { The Author(s). Published by S. Karger AG, Basel } \\
\text { www.karger.com/cpb }\end{array}$ \\
\hline
\end{tabular} \\ Zhou et al.: Microbiota in Non-Alcoholic Fatty Liver Disease}

In this study, we described the characteristics of gut microbiota and adiponectin in the progression of NAFLD. Our results suggest that both an imbalanced gut microbiota and adiponectin DNA methylation might be involved in the pathogenesis and progression of NAFLD. Probiotics therefore may delay the progression of NAFLD. This study assessed the effect of antibiotics and probiotics on NAFLD progression from the perspective of gut microecology and provides a theoretical foundation for targeting gut mircobiota in NAFLD management.

\section{Materials and Methods}

\section{Rat use and IACUC compliance}

Eight-week-old specific pathogen-free male SD rats were purchased from GDMLAC Laboratory (Guangdong Medical Laboratory Animal Center, Foshan, China). After 1 week of free access to a standard diet, 50 rats were randomly divided into five groups for 13 weeks: (A) Standard chow diet (SCD) (n = 10); (B) high fat diet (HFD) ( $n=10)$; (C) SCD with antibiotic (SCD-A) $(n=10)$; (D) HFD with antibiotics (HFD-A) $(n=10)$ and (E) HFD with antibiotics and probiotics (HFD-AP) $(n=10)$. The standard chow diet was comprised of $80 \%$ carbohydrates, $15 \%$ proteins, and 5\% fats, while the HDF diet contained $52.2 \%$ standard diet, $15 \%$ lard, $20 \%$ sucrose, $1.2 \%$ cholesterol, and $0.2 \%$ bile salt. All experimental rats had ad libitum access to food and water.

Rats were housed at $22-28^{\circ} \mathrm{C}$ and $60 \%$ relative humidity in a specific pathogen-free facility maintained on a 12-hour light/dark cycle in Guangzhou Medical University. The physical activity, emotional behavior, consumption of food and water, and defecation of experimental animals were observed daily.

For the antibiotic intervention, rats were orally administered $1 \mathrm{ml} 157.5 \mathrm{mg} / \mathrm{ml}$ ceftriaxone twice daily for the first four days and then the ceftriaxone dose was decreased to $0.35 \mathrm{mg} / \mathrm{ml}$ added to the drinking water for the remainder of the 13 week observation period. Probiotic treated, rats were administered a HFD diet concomitantly supplemented with probiotics $(0.35 \mathrm{~g} / \mathrm{kg} /$ day $)$ for 13 weeks by oral gavage. Probiotic supplementation consisted of $0.5 \times 10^{6}$ colony-forming units (CFU) live Bifidobacterium longum, Lactobacillus acidophilus, and Enterococcus faecalis.

The Animal Ethics Committee of Guangzhou Medical University approved all experimental protocols involving animals. All treatment procedures were performed in accordance with the guidelines of the Institutional Animal Care and Use Committee (IACUC) of Guangzhou Medical University. Animal treatments lasted for 13 weeks, during which the body weight and food intake of each animal was measured once a week.

\section{Animal euthanasia and tissue extraction}

At the end of prescribed feeding period, all rats were fasted overnight and anesthetized with an intraperitoneal injection of $10 \%$ chloral hydrate $(100 \mu \mathrm{l} / 20 \mathrm{~g}$ total body weight). Once rats were anaesthetized, blood samples were collected from the inferior vena cava. The liver and intestinal tissues were excised and weighed immediately. The liver index was calculated using the following formula liver wet weight/total body weight $\times 100 \%$.

\section{Pathology and related index detection}

The left lobe of the liver was immersed in 4\% neutral formaldehyde; Hematoxylin and Eosin (HE) and Masson staining were completed in the Guangzhou First People's Hospital, Guangzhou Medical University. HE staining and Masson staining of sections were performed using standard protocols.

Fasting venous blood samples were acquired in the morning and centrifuged at $3000 \mathrm{~g} / \mathrm{min}$ and $4{ }^{\circ} \mathrm{C}$ for $10 \mathrm{~min}$. Recovered supernatants were separated into $200 \mu \mathrm{l}$ tubes and immediately frozen at $-80^{\circ} \mathrm{C}$. Liver function indexes such as ALT, AST, triglyceride (TG), total cholesterol (TC), high-density lipoprotein (HDL-C), and low-density lipoprotein (D-LDL) were detected by the clinical laboratory of Guangzhou First People's Hospital of Guangzhou Medical University. Residual liver tissue was stored at $-80^{\circ} \mathrm{C}$. 


\section{Cellular Physiology Cell Physiol Biochem 2018;51:2123-2135 \begin{tabular}{l|l|l} 
and Biochemistry Published online: 6 December 2018 & $\begin{array}{l}\text { (c) } 2018 \text { The Author(s). Published by S. Karger AG, Basel } \\
\text { www.karger.com/cpb }\end{array}$ \\
\hline
\end{tabular}

\section{Serum adiponectin and liver DNA methylation measurements}

Serum adiponectin levels were measured by enzyme-linked immunosorbent assay (ELISA). The liver tissue DNA was isolated using the QIAamp DNA Mini Kit according to the standard protocol provided by the manufacturer (QIAGEN, Hilden, Germany). Concentration of extracted DNA was measured using a Nanodrop spectrophotometer 2000c (Thermo Scientific, USA). DNA after bisulfite modification (QIAGEN EpiTect Bisulfie kit) and purification was then amplified for the adiponection gene using the PCR kit (QIAGEN PyroMark kit) (Table S1 - for all supplemental material see www.karger.com/ 10.1159/000495830/). Amplified products were then separated by gel electrophoresis to obtain a single band. Those products with biotin labels were then mixed with microbeads carrying streptavidin. The single-stranded DNA with biotin labels was separated with unlabeled strands. Single-stranded DNA was then mixed with sequencing primers for adiponectin. The methylation status of the adiponectin gene promoter was quantified with a Pyromark Q96ID pyrophosphate sequencing analyzer (QIAGEN, Germany).

\section{S rRNA gene sequencing of gut microbial communities analysis}

Cecal DNA was isolated using the QIAamp DNA Mini Kit (QIAGEN, Hilden, Germany). The extracted DNA from each sample was used as the template to amplify the V4 region of 16S rRNA genes (Table S1). For 16S rRNA gene sequencing, DNA samples were send to the Beijing Genomic Institute (BGI, Shenzhen, China). PCR amplification, Illumina sequencing of the PCR amplicons (Illumina MiSeq platform) using the 250-bp paired-ended strategy and quality control of raw data were performed as described previously [22]. Sequencing results were clustered utilizing the barcoded Illumina paired-end (PE) sequencing (BIPES) process for preliminary analysis, while the remaining sequences were screened by UCHIME and suspected chimeric sequences were removed. All reads were sorted into different samples according to their barcodes. We picked a representative sequence for each OTU and used the RDP classifier [23] to annotate taxonomic information for each representative sequence. The closed-reference OTU clustering was done at $97 \%$ similarity level against the GreenGenes database (v201305) [24] and RDP Release9 201203 [25]. Principal component analysis (PCA) based on UniFrac distance was performed with QIIME. The linear discriminant analysis (LDA) with effect size measurements (LEfSe) was used to identify indicator bacterial groups specialized within the two groups.

\section{Statistical analysis}

Data are presented as mean \pm SD. Statistical significance for body weight, liver weight, liver index and serum index (such as AST, ALT, TG, TC, HDL-C, D-LDL, APN) were determined with one way analysis of variance (ANOVA) followed by Tukey's post hoc and Bonferroni multiple comparison test. The DNA methylation levels and gut microbiota data were analyzed by Kruskal-Wallis, followed by the Wilcoxon comparison tests for pairwise comparisons. A p-value $<0.05$ was considered statistically significant. Statistical analysis performed with SPSS software (Version 21, SPSS Inc., Chicago, USA).

\section{Results}

\section{Effects of antibiotics and probiotics on liver function}

The treatment course was completed with no animals becoming moribund. After feeding the rats with HFD, those in the antibiotic groups gradually displayed anorexia, fatigue, lethargy, and chills. The stool of HFD animals was much looser, and rats from antibiotic groups had soft, pulpy, and smelly feces. The body weight, liver wet weight, and liver index of rats were affected by HFD and gut microbiota (antibiotic and probiotic). Weekly body weight observations, demonstrated rats with HFD, HFD-A, and HFD-AP gained weight slower than rats treated with SCD and SCD-A (Fig. 1A). At the end of week 13, all rats fed by HFD were observably thinner than those fed by SCD and weekly body weights reflected this observation $(p<0.01)$. Liver wet weight and liver index were significantly increased among the HFD, HFD-A, and HFD-AP treatment groups ( $\mathrm{p}<0.01$ ) (Fig. 1B, 1C, 1D).

The serum ALT and AST levels reported liver function injury and TG, TC, HDL-C, and D-LDL reported complications with metabolic syndrome. Elevated ALT and AST values for the HFD, HFD-A, and HFD-AP groups indicated some degree of liver injury (Fig. 2A and 2B). 
Treatment groups HFD, HFD-A, and HFD-AP demonstrated increased TC and D-LDL (Fig. 2D and 2F) with no obvious change in either serum HDL and triglycerides (Fig. 2C and 2E).

\section{Effects of antibiotics and probiotics on hepatic phenotypes}

When rats received 13 weeks HFD intake, steatosis was induced with a greater severity among the HFD-A treatment group. Addition of the probiotic partly improved the liver steatosis outcome (Fig. 3A). HE staining showed that the HFD group displayed NAFLD changes with invasion of small vesicular fat and inflammatory cells. Moreover, the HFD-A group had apparent liver steatosis and neutrophil infiltration (Fig. 3B). HFD-AP animals a partial reversal of hepatocyte steatosis and neutrophil infiltration. Masson staining showed fibrous hyperplasia in the hepatic sinks of some rats in the HFD-A group and fiber spacing was observed around the sinks (Fig. 3C). Together these results support a suggested link between liver function and hepatic inflammatory cell infiltration as contributors to steatohepatitis development. Further, the ability to recover from steatosis in HFD mice, with antibiotic and probiotic intervention, suggests an associated link between gut microbiota and disease progression.

\section{Effects of antibiotics and probiotics on adiponectin and DNA methylation}

Adiponectin, a protein hormone secreted by adipose tissues, is of great importance to the development of NAFLD. With the addition of antibiotic management, serum adiponectin in HFD rats was decreased compared to SCD rats. When compared to SCD groups, HFD-A rats had significantly lower serum levels of adiponectin which was then observed to be partially recoverable with the addition of probiotics ( $p<0.05$ ) (Fig. 4A).

Furthermore, adiponectin DNA methylation is suggested to participate in the pathogenesis of NAFLD. In order to understand role of the intestinal microbiota, specifically alterations related to adiponectin DNA methylation, the methylation level of $10 \mathrm{CpG}$ island sites in the adiponectin promoter region were detected by pyrophosphate sequencing (Supplement 1). Interestingly, results showed obvious shifts in the degree of adiponectin

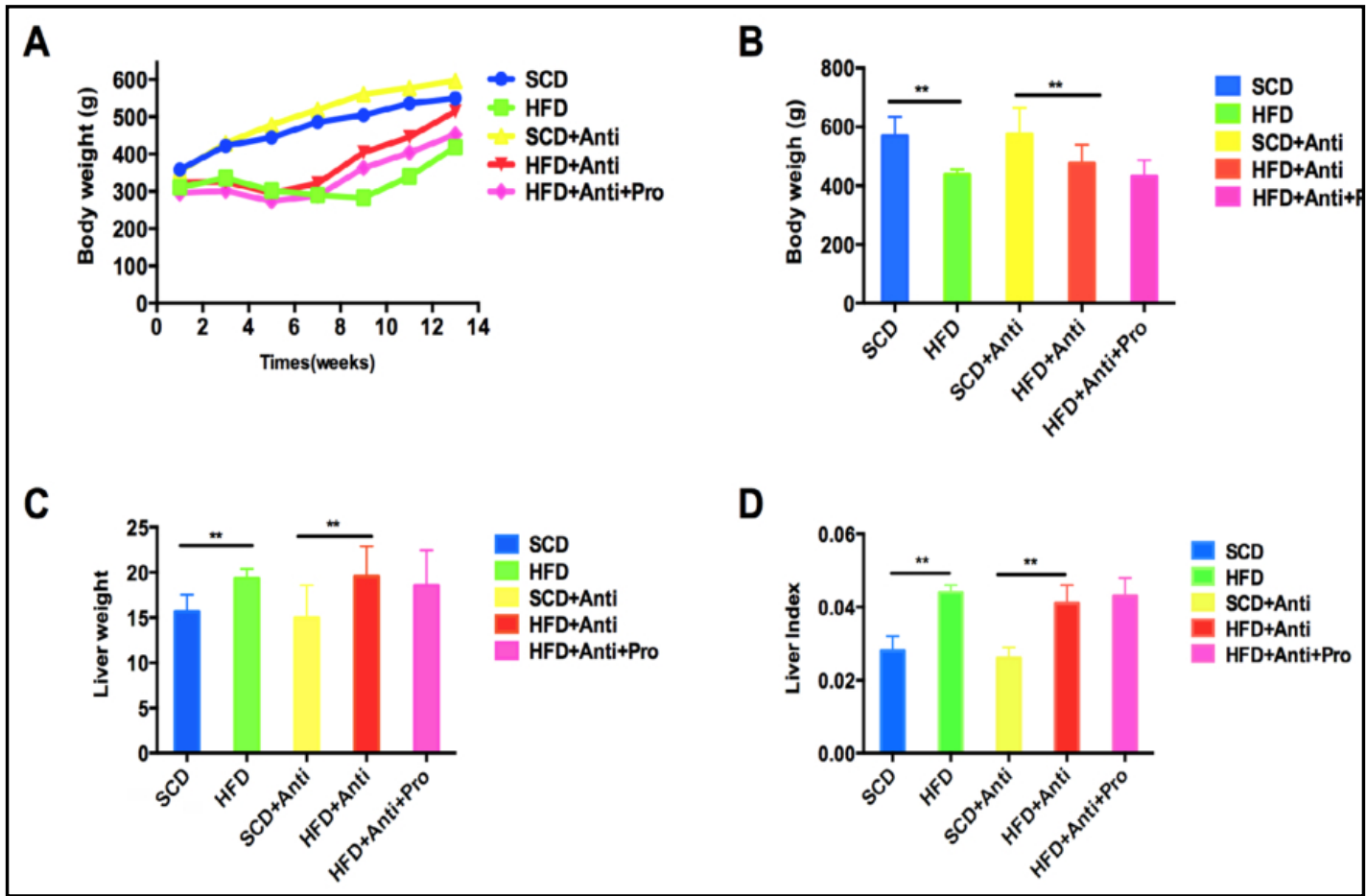

Fig. 1. Observations of (A and B) body weight, (C) liver weight, and (D) liver index of control and NAFLD rats across treatment groups $(\mathrm{n}=10)$ are displayed as the mean \pm SD of each group. ${ }^{*} \mathrm{p}<0.05 ;{ }^{* *} \mathrm{p}<0.01$. 


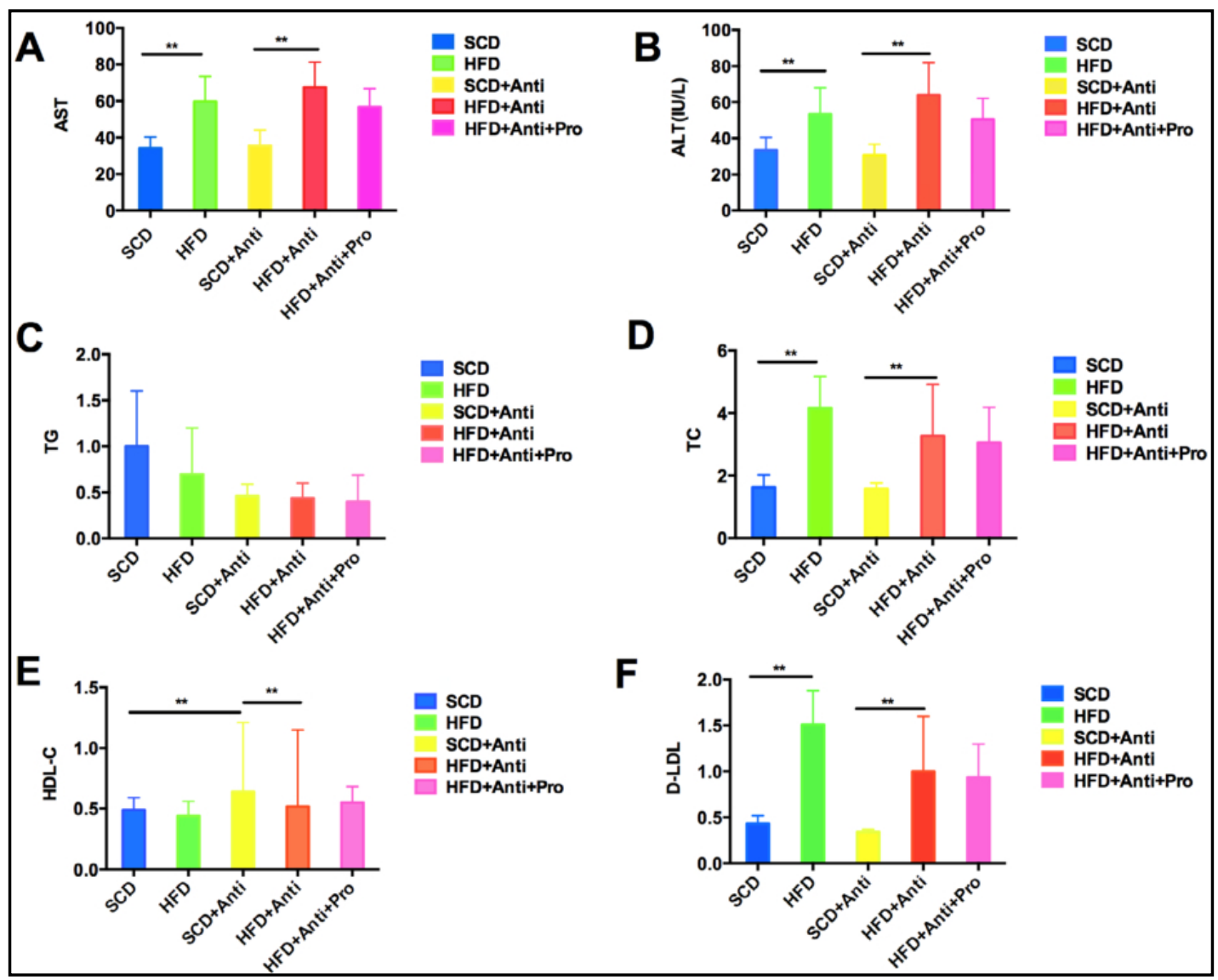

Fig. 2. Influence of antibiotic and probiotic exposure on blood biochemical indexes of HFD rats. (A) Serum aspartate aminotransferase (AST), (B) serum alanine aminotransferase (ALT), (C) serum TG, (D) serum TC, (E) serumhigh-densitylipoprotein (HDL-C) and (F) low-densitylipoprotein (D-LDL). Significance wasassessedby one-way ANOVA. Data are expressed as means \pm SD. ${ }^{*} \mathrm{p}<0.05$ and ${ }^{* *} \mathrm{p}<0.01$ across all treatment groups $(n=10)$.

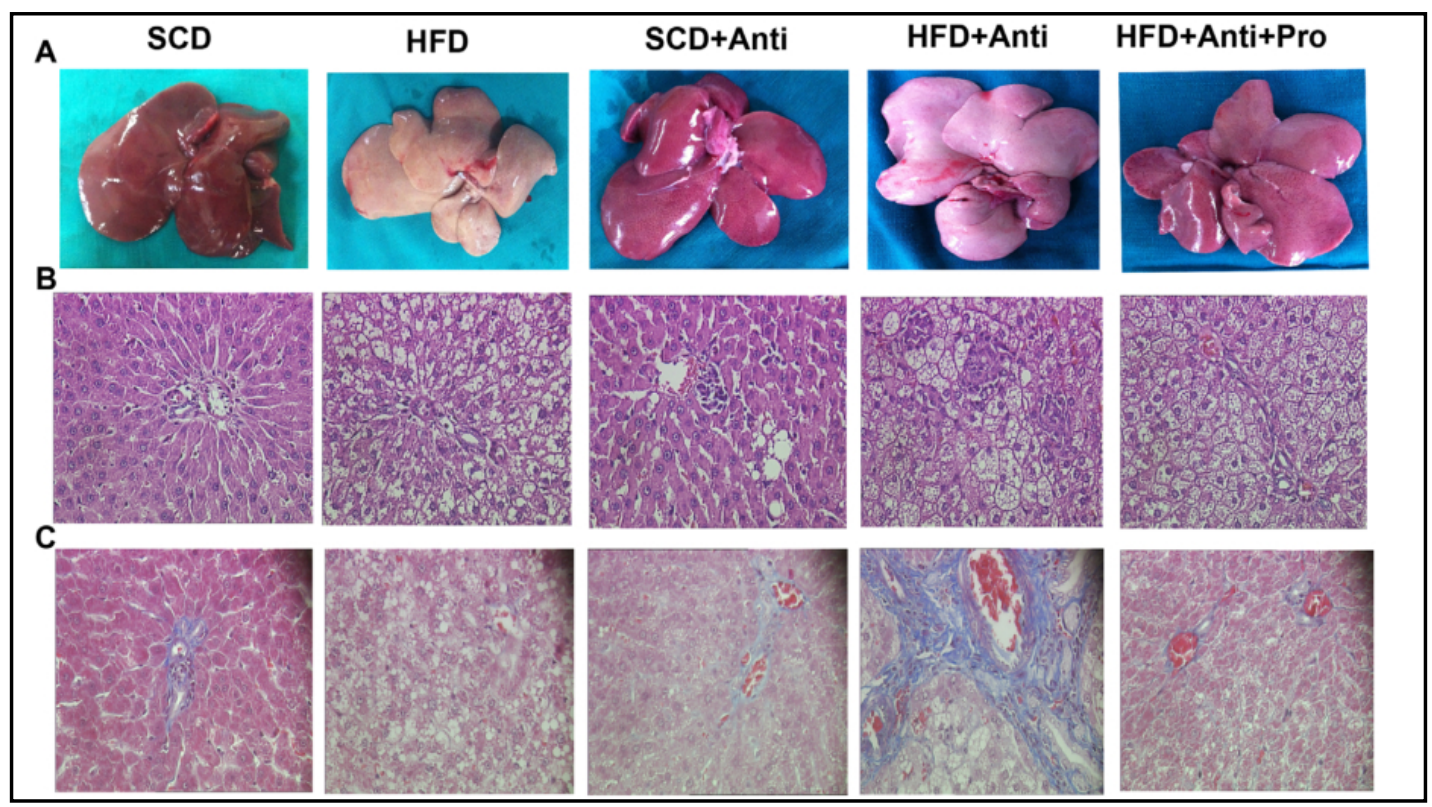

Fig. 3. Histology of controls and NAFLD models including: (A) Representative liver morphology, (B) hematoxylin and eosin, and (C) Masson stained liver sections. 
methylation in NAFLD rats with antibiotic intervention (HFD-A) compared to HFD only rats (HFD). At sites 2, 6, and 9, the methylation level in HFD-A rats was much higher than rats without antibiotics $(\mathrm{p}<0.05)$. No significant differences were observed across 7 other CpG sites (Fig. 4B, 4C, and 4D).

\section{Effects of antibiotics and probiotics on gut microbiota in NAFLD rats}

In order to evaluate the potential impact of alterations to the gut microbial community on the development of NAFLD, we treated rats with antibiotics and probiotics on a HFD for 13 weeks. To assess the effect of NAFLD on cecal microbial composition and the relative abundance of specific gut microbiota, targeted microbial 16S rRNA gene sequencing and bioinformatics based analysis were performed on cecal tissue samples collected at the end of the treatment in week 13 . A total number of $6,258,831$ high-quality tags were obtained from the 50 samples of the study, with an average of $127,731 \pm 25,592$ sequences per sample. 573 OTUs were shared across groups shown by Venn diagram (Fig. 5A). Clustering of sequences by unweighted UniFrac distances per rat (Principal component analysis, PCA) revealed a sharp clustering of microbiome sequence data among the HFD, HFD-A, and HFDAP treatment groups, indicating that following HFD and HFD with bacterial intervention treatments had distinct colonic microbial communities (Fig. 5B).

The probiotic treatment increased microbial alpha diversity, measured by the Shannon index (Fig. 5C). Firmicutes, Bacteroidetes, and Proteobacteria are the most abundant phyla, accounting for an average $95 \%$ of yielded sequences, while Verrucomicrobia, Cyanobacteria, and Deferribacteres accounted for $0.1 \%-5 \%$ of yielded sequences each (Fig. 5D, E and F). Genus level characterization is more complex and the 20 most abundant genera observed

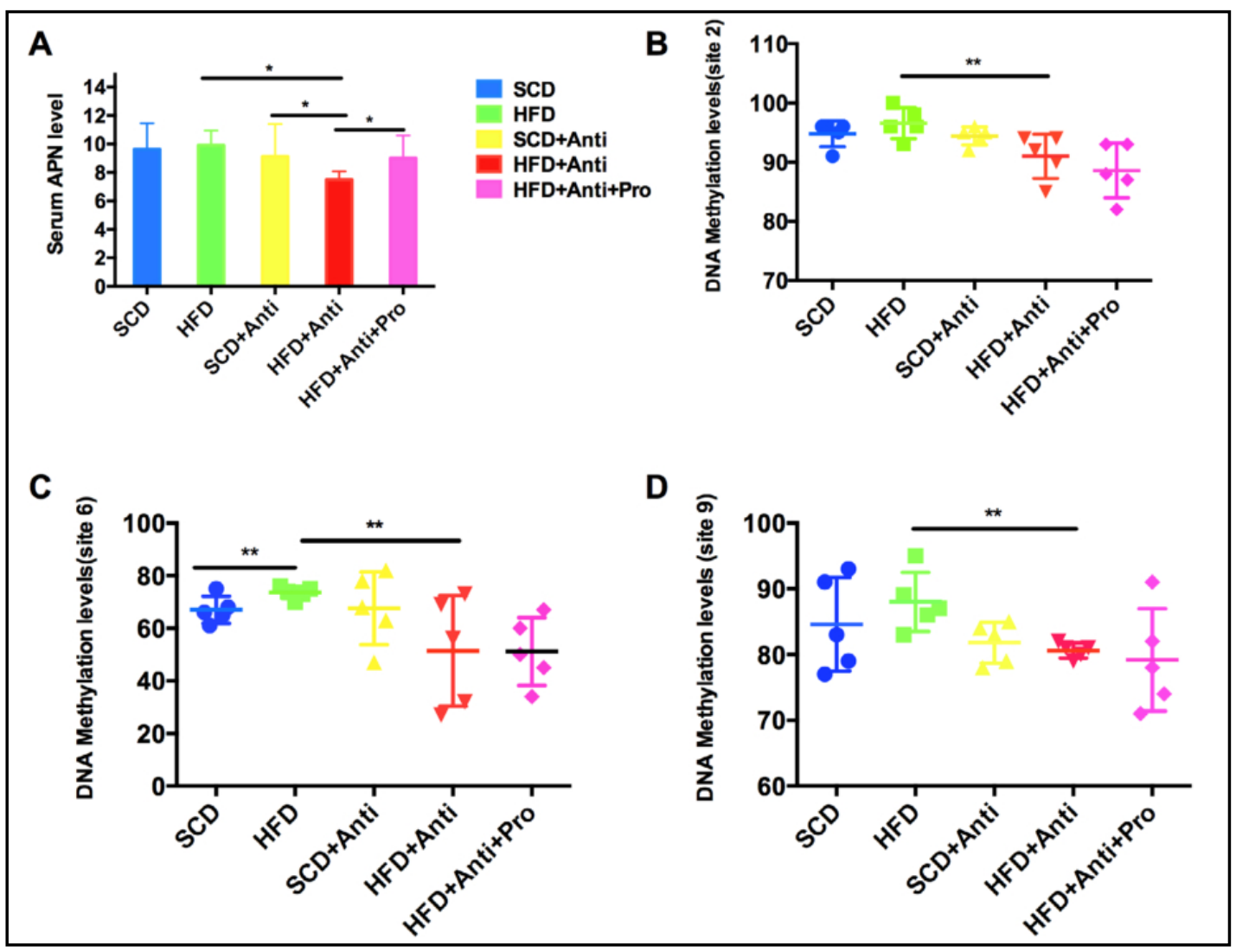

Fig. 4. Observations of serum adiponectin concentrations and liver adiponectin DNA methylation in control and NAFLD rats: (A.) Serum APN concentration determined by ELISA. (B.) Liver APN DNA methylation by pyrophosphate sequencing across treatment groups $(\mathrm{n}=5),{ }^{*} \mathrm{p}<0.05$ and ${ }^{* *} \mathrm{p}<0.01$ 


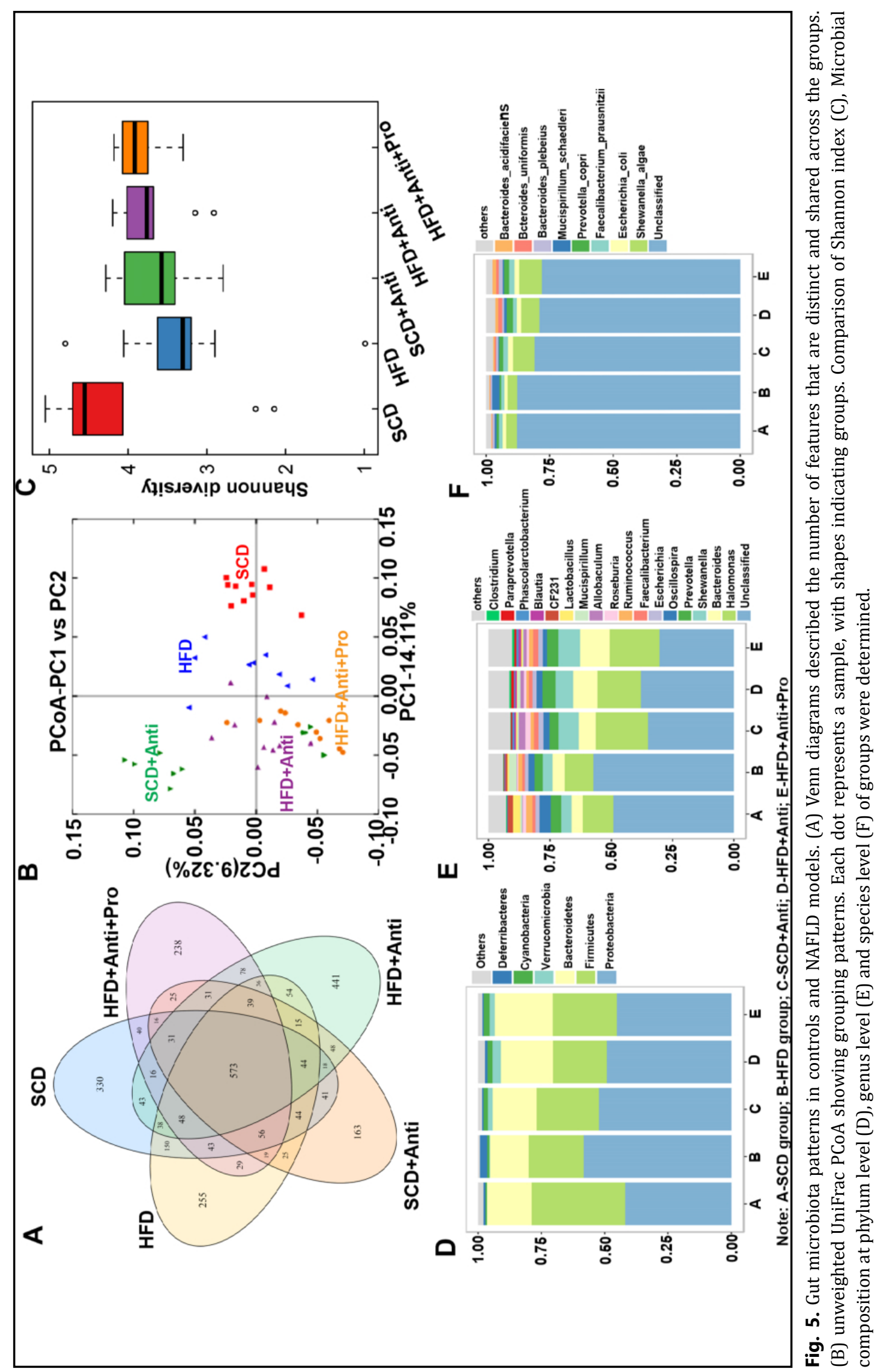


in our study only constituted $60 \%$ of the total microbiome, with Halomonas and Bacteroides dominating the composition (Fig. 5E). Together, HFD-A resulted in distinct gut microbiota alterations, reduced bacterial diversity, and an abundance of Firmicutes and Lactobacillus when compared to the HFD group. Increased composition abundance of

Planctomycetes, Gemmatimonadetes, Acidobacteriaceae, Acidobacteriales, Acidobacteria, Verrucomicrobiae, Verrucomicrobiales and Verrucomicrobiaceae were also observed following HFD-A; however HFD-AP was able to partly reverse the shift (Fig. 6).

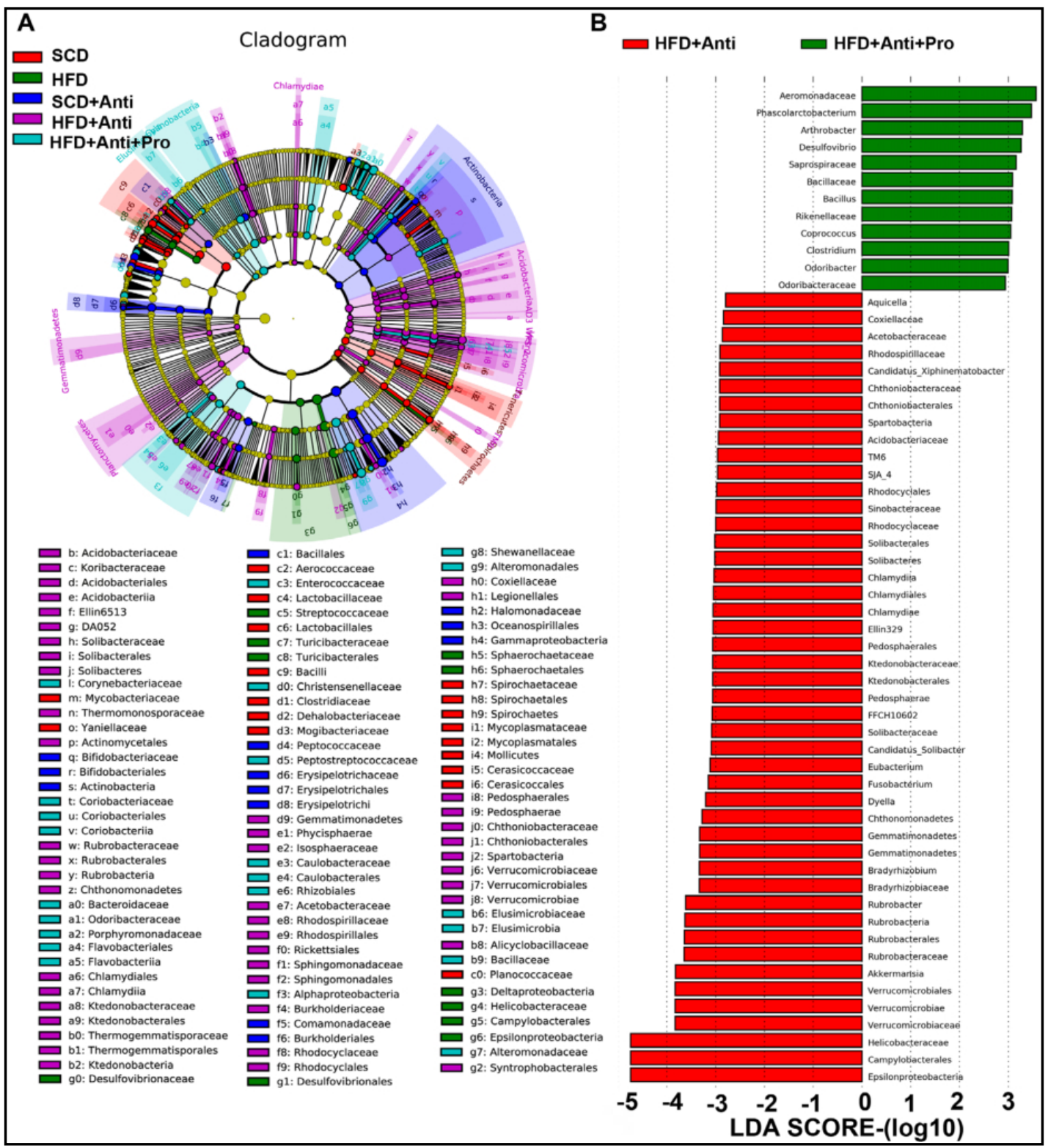

Fig. 6. Taxa listed according to their LDA values among groups (A) and between HFD with antibiotics and probiotics treatment group (B) computed from LEfSe. 


\section{Cellular Physiology Cell Physiol Biochem 2018;51:2123-2135 and Biochemistry Published online: 6 December $2018 \begin{aligned} & \text { DOI: } 2018 \text { The Author(s). Published by S. Karger AG, Basel } \\ & \text { www.karger.com/cpb }\end{aligned}$ \\ Zhou et al.: Microbiota in Non-Alcoholic Fatty Liver Disease}

\section{Discussion}

The gut microbiota has recently been acknowledged as an essential "organ" of the human body. Gaining an understanding of its roles and the way in which diet, exercise, stress, and antibiotic and probiotic use all can influence the microbial diversity and the bacterial gene function of the gut microbiome is essential [26]. Mouzaki, et al., reported a significant link between the presence of NASH and low percentages of Bacteroidetes in fecal samples, but not BMI, suggesting that intestinal flora may play an important role in the development of NAFLD [27]. An imbalanced gut flora could increase the production of endotoxins or even result in intestinal endotoxemia, which are important factors involved in secondary liver damage as well as the disease progression of NAFLD [28, 29]. In return, the development of NAFLD affects the intestinal micro-ecological balance, forming a vicious circle.

In the present study, serum adiponectin levels were determined by ELISA and methylation levels of $10 \mathrm{CpG}$ sites of adiponectin promoter regions were detected by pyrophosphate sequencing. Further, genomic DNA from the rat colonic samples were amplified with universal primers for the V4 region of bacterial 16S rRNA. Using Illumina sequencing techniques gut microflora diversity was detected and analyzed for gut microbiota characteristics in the progression of NAFLD in rats.

Adiponectin plays a central role in regulating insulin resistance, oxidative stress, and mitochondrial dysfunction in multiple organs [30]. A number of studies have shown that adiponectin is associated with the development of a fatty liver and increased plasma, adiponectin and its derivatives can also increase liver tissue damage and steatosis [31]. In vivo, persistent high blood insulin is negatively correlated with low adiponectin levels [30]. NAFLD patients often exhibit low adiponectin when insulin resistance is present [32]. In addition, adiponectin could inhibit the progression of liver inflammation and fibrosis by inhibiting the synthesis and release of TNF-alpha [33]. Serum concentrations of adiponectin in the HFD-A group were significantly decreased and marginally recoverable with the addition of probiotics, as suggested by HFD-AP data. Together these data suggest serum APN levels are directly and inversely related the progressive variability of liver pathology and are potentially linked to intestinal microbiota shifts. adiponectin and intestinal flora are known to play an important role in the development of NAFLD; therefore probiotic intervention may be of great clinical value when considering potentially solving the major challenge of managing NAFLD in a clinical setting.

Recently increasing efforts have been focused on the mechanisms driving gut microecology. Modulation of gut microbiota with probiotics, prebiotics, or fecal microbiota transplantation (FMT) have recently been implemented in the prevention and treatment of several metabolic diseases, such as NAFLD [34]. Previous data suggests that manipulation of the gut microbiota may yield novel therapies for the management of NAFLD [20]. Modulating gut flora with diet or therapeutic agents has been suggested to improve host metabolic phenotypes $[35,36]$; however, whether the response of the gut microbiota to these environmental perturbations happens at the phylum or specific phylotype level remains controversial [37]. Genus Akkermansia of Verrucomicrobia is involved in mucus degradation but may also have beneficial effects via increasing intestinal levels of endocannabinoids that improve inflammation and gut barrier function [38]. Turnbaugh, et al. observed that the frequency of increased Firmucutes species coinciding with decreased Bacteriodetes in the guts of obese mice with an elevated colonic fermentation and SCFA production potentially contributing to obesity by increasing the host's capacity for energy harvesting from foods [38]. Apart from effectively reducing fat mass and body weight, calorie restriction markedly increased the intestinal microbial diversity, specifically Allobaculum abundance, in HFD mice but not in those receiving SCD [39]. In the present study, Faecalibacterium prausnitzii and Blautia, which were short-chain fatty acid (SCFA) producers, decreased in HFD rats and could be reversed by the addition of a probiotic treatment. Intervention with probiotics has the potential to inhibit the proliferation of aerobic bacterial species while upregulating the proportion of anaerobic bacteria and gut flora colonization resistance. These results suggest

\section{KARGER}




\section{Cellular Physiology Cell Physiol Biochem 2018;51:2123-2135

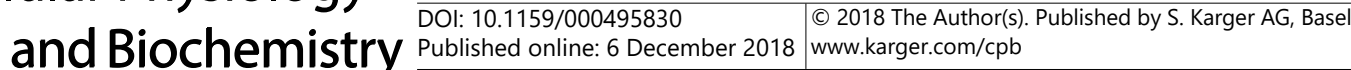 \\ Zhou et al.: Microbiota in Non-Alcoholic Fatty Liver Disease}

that probiotic administration could enrich the relative abundance of SCFA producers to promote colonic fermentation and SCFA production in the guts of HFD-fed rats [40, 41]. Our findings were consistent with studies demonstrating that probiotics could improve the structure and proportion of the gut microbiota and slow the progression of liver injury in NAFLD [42].

\section{Conclusion}

In summary, probiotics could improve gut microbial imbalance presented in NAFLD and restore the microecosystem of intestinal microbiota via up regulation of adiponectin. Therefore probiotics may reduce hepatic and systemic pro-inflammatory responses caused by adiponectin DNA methylation signaling. Consequently, probiotics could ameliorate liver inflammation and thus delay or prevent NAFLD progression. Our findings have revealed that probiotic supplementation can realize the balance of gut microbiota, suggesting bacterial intervention targeting gut microbiota as a viable novel approach to prevention and treatment of NAFLD.

\section{Acknowledgements}

This work was supported by the grants from the National Natural Science Foundation of China (NSFC 81700487, NSFC81871905), the Fundamental Research Funds for the Central Universities (Grant No. 2018MS82 by Youlian Zhou) and the Guangzhou Technology Projects (No. 2019-03-01-04-2024-0004, Yongjian Zhou).

The Animal Ethics Committee of Guangzhou Medical University approved all experimental protocols involving animals. All treatment procedures were performed in accordance with the guidelines of the Institutional Animal Care and Use Committee (IACUC) of Guangzhou Medical University.

Author Contributions: ZYL: statistical analysis and interpretation of the data, drafting of the article; ZTY: conduction of the experiments, and interpretation of the data, revision of the article; CHT and LYQ: analysis and interpretation of the data, revision of the article; HHL, CWJ and DYL: interpretation of the data and revision of the article; HJ and LYY: interpretation of the data; CJ and NYQ: revision of the article; ZYJ: concept and design of the study, interpretation of the data and revision of the article.

\section{Disclosure Statement}

The authors declare that no conflict of interest exists.

\section{References}

$\checkmark 1$ Cohen JC, Horton JD, Hobbs HH: Human fatty liver disease: old questions and new insights. Science 2011;332:1519-1523.

-2 Ahmed M: Non-alcoholic fatty liver disease in 2015. World J Hepatol 2015;7:1450-1459.

3 Milic S, Stimac D: Nonalcoholic fatty liver disease/steatohepatitis: epidemiology, pathogenesis, clinical presentation and treatment. Dig Dis 2012;30:158-162.

4 Williams CD, Stengel J, Asike MI, Torres DM, Shaw J, Contreras M, Landt CL, Harrison SA: Prevalence of nonalcoholic fatty liver disease and nonalcoholic steatohepatitis among a largely middle-aged population utilizing ultrasound and liver biopsy: a prospective study. Gastroenterology 2011;140:124-131.

5 Bhala N, Younes R, Bugianesi E: Epidemiology and natural history of patients with NAFLD. Curr Pharm Des 2013;19:5169-5176. 


\section{Cellular Physiology Cell Physiol Biochem 2018;51:2123-2135 and Biochemistry \begin{tabular}{l|l|l|l} 
DOI: 10.1159/000495830 & $\begin{array}{l}\text { ○ } 2018 \text { The Author(s). Published by S. Karger AG, Basel } \\
\text { www.karger.com/cpb }\end{array}$
\end{tabular}

6 Yoon HJ, Cha BS: Pathogenesis and therapeutic approaches for non-alcoholic fatty liver disease. World J Hepatol 2014;6:800-811.

7 Bettermann K, Hohensee T, Haybaeck J: Steatosis and steatohepatitis: complex disorders. Int J Mol Sci 2014;15:9924-9944.

-8 Starley BQ, Calcagno CJ, Harrison SA: Nonalcoholic fatty liver disease and hepatocellular carcinoma: a weighty connection. Hepatology 2010;51:1820-1832.

9 Than NN, Newsome PN: A concise review of non-alcoholic fatty liver disease. Atherosclerosis 2015;239:192-202.

10 Dowman JK, Tomlinson JW, Newsome PN: Pathogenesis of non-alcoholic fatty liver disease. QJM 2010;103:71-83.

-11 Zhou YJ, Li YY, Nie YQ Yang H, Zhan Q, Huang J, Shi SL, Lai XB, Huang HL: Influence of polygenetic polymorphisms on the susceptibility to non-alcoholic fatty liver disease of Chinese people. J Gastroenterol Hepatol 2010;25:772-777.

12 Hui Y, Yu-Yuan L, Yu-Qiang N, Wei-Hong S, Yan-Lei D, Xiao-Bo L, Yong-Jian Z: Effect of peroxisome proliferator-activated receptors-gamma and co-activator-1alpha genetic polymorphisms on plasma adiponectin levels and susceptibility of non-alcoholic fatty liver disease in Chinese people. Liver Int 2008;28:385-392.

13 Turek-Plewa J, Jagodzinski PP: The role of mammalian DNA methyltransferases in the regulation of gene expression. Cell Mol Biol Lett 2005;10:631-647.

-14 Bouchard L, Hivert MF, Guay SP, St-Pierre J, Perron P, Brisson D: Placental adiponectin gene DNA methylation levels are associated with mothers' blood glucose concentration. Diabetes 2012;61:12721280.

15 Shimizu K, Onishi M, Sugata E, Sokuza Y, Mori C, Nishikawa T, Honoki K, Tsujiuchi T: Disturbance of DNA methylation patterns in the early phase of hepatocarcinogenesis induced by a choline-deficient L-amino acid-defined diet in rats. Cancer Sci 2007;98:1318-1322.

-16 Qin J, Li R, Raes J, Arumugam M, Burgdorf KS, Manichanh C, Nielsen T, Pons N, Levenez F, Yamada T, Mende DR, Li J, Xu J, Li S, Li D, Cao J, Wang B, Liang H, Zheng H, Xie Y et al.: A human gut microbial gene catalogue established by metagenomic sequencing. Nature 2010;464:59-65.

17 Wieland A, Frank DN, Harnke B, Bambha K: Systematic review: microbial dysbiosis and nonalcoholic fatty liver disease. Aliment Pharmacol Ther 2015;42:1051-1063.

18 Tremaroli V, Backhed F: Functional interactions between the gut microbiota and host metabolism. Nature 2012;489:242-249.

19 Compare D, Coccoli P, Rocco A, Nardone OM, De Maria S, Carteni M, Nardone G: Gut--liver axis: the impact of gut microbiota on non alcoholic fatty liver disease. Nutr Metab Cardiovasc Dis 2012;22:471-476.

20 Li Z, Yang S, Lin H, Huang J, Watkins PA, Moser AB, Desimone C, Song XY, Diehl AM: Probiotics and antibodies to TNF inhibit inflammatory activity and improve nonalcoholic fatty liver disease. Hepatology 2003;37:343-350.

-21 Vahamiko S, Laiho A, Lund R, Isolauri E, Salminen S, Laitinen K: The impact of probiotic supplementation during pregnancy on DNA methylation of obesity-related genes in mothers and their children. Eur J Nutr 2018;10.1007/s00394-017-1601-1.

22 Zhou HW, Li DF, Tam NF, Jiang XT, Zhang H, Sheng HF, Qin J, Liu X, Zou F: BIPES, a cost-effective highthroughput method for assessing microbial diversity. ISME J 2011;5:741-749.

23 Wang Q, Garrity GM, Tiedje JM, Cole JR: Naive Bayesian classifier for rapid assignment of rRNA sequences into the new bacterial taxonomy. Appl Environ Microbiol 2007;73:5261-5267.

24 DeSantis TZ, Hugenholtz P, Larsen N, Rojas M, Brodie EL, Keller K, Huber T, Dalevi D, Hu P, Andersen GL: Greengenes, a chimera-checked 16S rRNA gene database and workbench compatible with ARB. Appl Environ Microbiol 2006;72:5069-5072.

-25 Cole JR, Wang Q, Fish JA, Chai B, McGarrell DM, Sun Y, Brown CT, Porras-Alfaro A, Kuske CR, Tiedje JM: Ribosomal Database Project: data and tools for high throughput rRNA analysis. Nucleic Acids Res 2014;42:D633-642.

26 Crawford PA, Crowley JR, Sambandam N, Muegge BD, Costello EK, Hamady M, Knight R, Gordon JI: Regulation of myocardial ketone body metabolism by the gut microbiota during nutrient deprivation. Proc Natl Acad Sci U S A 2009;106:11276-11281. 


\section{Cellular Physiology Cell Physiol Biochem 2018;51:2123-2135 and Biochemistry \begin{tabular}{l|l|l} 
DOI: 10.1159/000495830 \\
Published 2018 The Author(s). Published by S. Karger AG, Basel
\end{tabular}

27 Mouzaki M, Comelli EM, Arendt BM, Bonengel J, Fung SK, Fischer SE, McGilvray ID, Allard JP: Intestinal microbiota in patients with nonalcoholic fatty liver disease. Hepatology 2013;58:120-127.

28 Feng Q Liu W, Baker SS, Li H, Chen C, Liu Q Tang S, Guan L, Tsompana M, Kozielski R, Baker RD, Peng J, Liu P, Zhu R, Hu Y, Zhu L: Multi-targeting therapeutic mechanisms of the Chinese herbal medicine QHD in the treatment of non-alcoholic fatty liver disease. Oncotarget 2017;8:27820-27838.

-29 Yuan J, Baker SS, Liu W, Alkhouri R, Baker RD, Xie J, Ji G, Zhu L: Endotoxemia unrequired in the pathogenesis of pediatric nonalcoholic steatohepatitis. J Gastroenterol Hepatol 2014;29:1292-1298.

-30 Calton EK, Miller VS, Soares MJ: Factors determining the risk of the metabolic syndrome: is there a central role for adiponectin? Eur J Clin Nutr 2013;67:485-491.

-31 Buechler C, Wanninger J, Neumeier M: Adiponectin, a key adipokine in obesity related liver diseases. World J Gastroenterol 2011;17:2801-2811.

32 Mirza MS: Obesity, Visceral Fat, and NAFLD: Querying the Role of Adipokines in the Progression of Nonalcoholic Fatty Liver Disease. ISRN Gastroenterol 2011;2011:592404.

-33 Asano T, Watanabe K, Kubota N, Gunji T, Omata M, Kadowaki T, Ohnishi S: Adiponectin knockout mice on high fat diet develop fibrosing steatohepatitis. J Gastroenterol Hepatol 2009;24:1669-1676.

34 Lau E, Carvalho D, Freitas P: Gut Microbiota: Association with NAFLD and Metabolic Disturbances. Biomed Res Int 2015;2015:979515.

35 Ley RE, Turnbaugh PJ, Klein S, Gordon JI: Microbial ecology: human gut microbes associated with obesity. Nature 2006;444:1022-1023.

36 Xie W, Gu D, Li J, Cui K, Zhang Y: Effects and action mechanisms of berberine and Rhizoma coptidis on gut microbes and obesity in high-fat diet-fed C57BL/6J mice. PLoS One 2011;6:e24520.

-37 Duncan SH, Lobley GE, Holtrop G, Ince J, Johnstone AM, Louis P, Flint HJ: Human colonic microbiota associated with diet, obesity and weight loss. Int J Obes (Lond) 2008;32:1720-1724.

-38 Turnbaugh PJ, Ley RE, Mahowald MA, Magrini V, Mardis ER, Gordon JI: An obesity-associated gut microbiome with increased capacity for energy harvest. Nature 2006;444:1027-1031.

-39 Ravussin Y, Koren O, Spor A, LeDuc C, Gutman R, Stombaugh J, Knight R, Ley RE, Leibel RL: Responses of gut microbiota to diet composition and weight loss in lean and obese mice. Obesity (Silver Spring) 2012;20:738-747.

40 Zhu L, Baker RD, Baker SS: Gut microbiome and nonalcoholic fatty liver diseases. Pediatr Res 2015;77:245251.

41 Jiao N, Baker SS, Nugent CA, Tsompana M, Cai L, Wang Y, Buck MJ, Genco RJ, Baker RD, Zhu R, Zhu L: Gut microbiome may contribute to insulin resistance and systemic inflammation in obese rodents: a metaanalysis. Physiol Genomics 2018;50:244-254.

42 de Moreno de LeBlanc A, LeBlanc JG: Effect of probiotic administration on the intestinal microbiota, current knowledge and potential applications. World J Gastroenterol 2014;20:16518-16528. 